This item was submitted to Loughborough's Research Repository by the author.

Items in Figshare are protected by copyright, with all rights reserved, unless otherwise indicated.

\title{
A cluster randomized controlled trial of strategies to increase adolescents' physical activity and motivation in physical education: results of the Motivating Active Learning in Physical Education (MALP) trial
}

\section{PLEASE CITE THE PUBLISHED VERSION}

http://dx.doi.org/10.1016/j.ypmed.2013.09.003

\section{PUBLISHER}

(c) Elsevier Inc.

\section{VERSION}

AM (Accepted Manuscript)

\section{LICENCE}

CC BY-NC-ND 4.0

\section{REPOSITORY RECORD}

Lonsdale, Chris C., Richard R. Rosenkranz, Taren Sanders, Louisa R. Peralta, Andrew Bennie, Ben Jackson, Ian M. Taylor, and David R. Lubans. 2019. "A Cluster Randomized Controlled Trial of Strategies to Increase Adolescents' Physical Activity and Motivation in Physical Education: Results of the Motivating Active Learning in Physical Education (MALP) Trial". figshare. https://hdl.handle.net/2134/15377. 
This item was submitted to Loughborough's Institutional Repository (https://dspace.lboro.ac.uk/) by the author and is made available under the following Creative Commons Licence conditions.

\section{creative
commons}

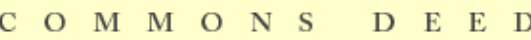

Attribution-NonCommercial-NoDerivs 2.5

You are free:

- to copy, distribute, display, and perform the work

Under the following conditions:

Attribution. You must attribute the work in the manner specified b the author or licensor.

Noncommercial. You may not use this work for commercial purposes.

No Derivative Works. You may not alter, transform, or build upon this work.

- For any reuse or distribution, you must make clear to others the license terms of this work.

- Any of these conditions can be waived if you get permission from the copyright holder.

Your fair use and other rights are in no way affected by the above.

This is a human-readable summary of the Leqal Code (the full license).

\section{Disclaimer 만}

For the full text of this licence, please go to: http://creativecommons.org/licenses/by-nc-nd/2.5/ 
Paper Title

A Cluster Randomized Controlled Trial of Strategies to Increase Adolescents' Physical Activity and Motivation in Physical Education: Results of the Motivating Active Learning in Physical Education (MALP) Trial

Authors

1. Chris Lonsdale

2. Richard R. Rosenkranz

3. Taren Sanders

4. Louisa Peralta

5. Andrew Bennie

6. Ben Jackson

7. Ian M. Taylor

8. David R. Lubans

\section{Author Institutes}

1. University of Western Sydney

2. Kansas State University \& University of Western Sydney

3. University of Western Sydney

4. University of Sydney

5. University of Western Sydney

6. University of Western Australia

7. University of Loughborough

8. University of Newcastle

Corresponding Author:

Chris Lonsdale

Email: c.lonsdale@uws.edu.au

Abstract Word Count: 200

Main Text Word Count: approximately 3400 (not including in-text citations) 


\begin{abstract}
Objective: Physical education (PE) programs aim to promote physical activity (PA) and reach most school-aged youth. However, PA levels within PE lessons are often low. In this clusterrandomized controlled trial, we examine the effects of three self-determination theory-based motivational strategies on PA and sedentary behavior, as well as their hypothesized antecedents during PE lessons.

Methods: Data were collected in Sydney, Australia (October - December 2011). After baseline testing, teachers $(n=16)$ and their classes $(n=288$ students; $M=13.6$ years, 50.4\% male) were randomly assigned to one of four teaching strategy conditions: (1) explaining relevance; (2) providing choice; (3) complete free choice; or (4) usual practice. Teachers then delivered the assigned strategy. Primary outcomes were accelerometer-assessed PA and student motivation during lessons. Secondary outcomes included sedentary behavior, perceptions of teachers' support and psychological needs satisfaction.

Results: The 'free choice' intervention increased PA $(p<.05)$. 'Providing choice' and 'free choice' interventions decreased sedentary behavior $(p<.05)$. The interventions did not influence motivation, but students’ autonomy increased during both choice-based interventions $(p<.05)$.

Conclusions: Promoting choice can produce short-term increases in PA and decreases in sedentary behavior, as well as increased perceived autonomy during PE lessons.
\end{abstract}

Keywords: self-determination theory; sedentary behavior; autonomy support; intervention; health behavior 


\section{Introduction}

Despite the extensive health benefits associated with physical activity (PA) (US Department of Health and Human Services, 2008), many children and adolescents are not active enough to achieve these positive effects (Hardy et al., 2010). Governmentcommissioned reports and policy statements from numerous countries underscore the important role that physical education (PE) programs can play in increasing youths' PA at a population level (Centers for Disease Control and Prevention, 2010; Crawford, 2009; Office for Standards in Education Children's Services and Skills, 2013). Indeed, the majority of school-aged children and adolescents attend regular PE lessons, and well-structured lessons can provide substantial opportunities for moderate-to-vigorous physical activity (MVPA). However, students' MVPA levels in PE lessons are often very low (Fairclough and Stratton, 2005; Fairclough and Stratton, 2006; Lonsdale et al., 2013; Office for Standards in Education Children’s Services and Skills, 2013).

To increase students' PA levels both during and outside of PE, it is important they are sufficiently and appropriately motivated (Aelterman et al., 2012; Hagger et al., 2005; Jaakkola et al., 2008). PE teachers can play an important role in motivating students (Cheon et al., 2012); therefore, it is important to examine strategies PE teachers can employ to motivate their students to engage in higher levels of PA.

\section{Theoretical Framework}

According to Self-Determination Theory (SDT), social-contextual factors, such as teaching strategies used by PE teachers, can affect motivation (Gillison et al., 2013; Ryan and Deci, 2002; Vansteenkiste et al., 2004). Recent SDT-based interventions have successfully enhanced student motivation towards PE (Chatzisarantis and Hagger, 2008; Cheon et al., 2012; Perlman, 2013; Ward et al., 2008); however, there is less evidence that SDT-based interventions in PE can influence actual MVPA behavior. In particular, there is a dearth of evidence gathered using objective MVPA measures (e.g., accelerometers) (Perlman, 2013). Also, it is unknown which teaching strategies involved in these SDT-based interventions are most effective in enhancing motivation and promoting MVPA. For example, explaining the relevance of activities and providing opportunities for students to make choices during lessons are considered important strategies to promote autonomous student motivation and increase MVPA (Cheon et al., 2012; Perlman, 2013). But, evidence regarding the specific effects of providing choice on motivation towards PE and MVPA during PE lessons is limited (Lonsdale et al., 2009; Ward et al., 2008) and no study has examined the effects of 
explaining relevance on these outcomes. Little experimental evidence from other domains exists, but correlational analyses suggest that relevance may be more important than choice in terms of students’ motivation towards academic subjects (Assor et al., 2002).

In the present study, we examined the independent effects of: (1) 'relevance'; (2) 'providing choice'; and (3) 'free choice'. Understanding which strategies have the greatest impact on motivation and MVPA may lead to more effective interventions to increase shortand long-term student motivation and MVPA, both during PE lessons and beyond.

Compared with usual practice, we hypothesized that PE lessons in which teachers explained the relevance of activities or provided students with opportunities to make choices would:

1. show greater increase in the proportion of PE lesson time students spend in MVPA.

2. show greater decrease in the proportion of PE lesson time that students spend in sedentary behavior.

3. show greater increase in students' autonomous motivation during lessons.

4. show greater decrease in students' controlled motivation during lessons.

5. show greater increase in students' perceptions of autonomy, competence, and relatedness during lessons.

6. show greater increase in students' and independent raters' perceptions of teachers' autonomy supportive behavior.

\section{Methods}

\section{Study Design}

This was a cluster-randomized controlled trial, registered with Current Controlled Trials and is traceable as ISRCTN07038258. The complete study protocol has been described elsewhere (Rosenkranz et al., 2012).

\section{Participants and Procedures}

The study was conducted in two Independent and three Catholic secondary schools in Sydney, Australia, with all data collected between October and December 2011. A university human research ethics committee provided ethical approval for this study. All school principals, PE teachers, and parents of participating students provided written consent, and 
students provided written assent. Students were eligible to participate in the study if they were physically able to participate in their PE lessons. Data were collected at baseline (i.e., before the intervention) and post-intervention ( $M=10.2, S D=6.9$ days after baseline).

\section{Interventions}

Teachers and their PE classes (1 class per teacher) were randomly assigned to a usual practice control arm or one of three intervention arms: (1) 'Relevance' ( $n=4$ classes) explaining the rationale and importance of the activity to the students' lives. Teachers were provided with example 'relevance' statements, including ways to help students see the connection between a skill development activity and a subsequent game and the possible social opportunities that students could enjoy through participation in an activity outside school. (2) 'Providing choice' ( $\mathrm{n}=4$ classes) - providing students with 2-4 opportunities for choice within the lesson (Patall et al., 2008). Teachers were provided with examples, such as providing students with a number of options for warm up activities and organizing two games near the end of the lesson -one 'competitive' game in which the score was kept, and one 'social' game in which the score was not recorded. (3) 'Free choice' ( $\mathrm{n}=4$ classes) providing the students with equipment, but refraining from giving instructions. Each teacher met with the principal investigator for 20 minutes to learn and plan the implementation of the teaching strategy in the post-intervention lesson. Teachers in the control arm ( $n=4$ classes) were instructed to continue with their usual teaching practice.

To test the fidelity of the interventions, teachers' verbal communication was recorded in the baseline and post-intervention lessons using a wireless recording device. Two independent researchers, blinded to arm allocation and study hypotheses, assessed teachers' autonomy supportive behaviors using items from the Teacher as Social Context Questionnaire (Taylor and Lonsdale, 2010). Scores on two items: (1) “The teacher gave the students choices about how they do the tasks in PE” (labeled 'choice provided'); and (2) "The teacher talks about how the students can use the things they learn in PE” (labeled 'relevance explained') were used to determine if the interventions had successfully manipulated choice and relevance, respectively. Both items included 7-point Likert ratings scales ( $1=$ Not at all true, $7=$ Very true). A random selection of $50 \%$ of the 32 lessons (baseline and postintervention) were double-coded, so that inter-rater reliability could be examined; intra-class correlations (ICCs) were acceptable (.95 and .88 for 'choice provided' and 'relevance explained' items, respectively). 


\section{Outcomes}

To control for differing duration of PE lessons, PA data (i.e., proportion of time spent in MVPA) were collected during the first 20 minutes of teaching time (i.e., after students had changed their clothes) in the baseline and post-intervention lessons. All PA data were measured using an Actigraph GT3X accelerometer (Actigraph; Pensacola, FL). Complete accelerometer procedure details can be viewed elsewhere (Rosenkranz et al., 2012).

At the conclusion of the 20-minute lessons, students completed questionnaires to measure their motivation towards the PE lesson (primary outcome) (Guay et al., 2000). Additionally, students completed questionnaires to measure their perceived autonomy (Ng et al., 2011; Standage and Gillison, 2007), competence (McAuley et al., 1989), and relatedness (Richer and Vallerand, 1998), as well as perceptions of teacher autonomy supportive behavior (Taylor and Lonsdale, 2010) (secondary outcomes). An overall self-determination index (SDI) was formed using scores from the four subscales of the Situational Motivation Scale (2* intrinsic motivation + identified motivation - external regulation $-2 *$ amotivation) (Lonsdale et al., 2009). Higher SDI scores indicate higher self-determination.

\section{Sample Size}

Based on Lonsdale et al. (2009)'s findings, we conservatively estimated a moderate effect size (medium $f=0.25$ ) for mixed (between and within-subjects) analysis. Using an alpha of .05, an estimated correlation of $r=.5$ (Lonsdale et al., 2009), and an anticipated intra-class correlation coefficient (ICC) of 0.1 (Campbell et al., 2004), we expected that a sample of 258 students was required for 95\% power. This sample size was then increased by $20 \%$, to allow for participant attrition; therefore, we aimed to recruit 308 students from $16 \mathrm{PE}$ classes across the five schools.

\section{Randomization}

After baseline assessments, an independent researcher, blinded to study hypotheses, used a computerized random number generator with blocked randomization scheme (block size $=4$ ) to assign classes to conditions. Three of the five schools provided four classes each and, therefore, each school constituted a block, with each class randomly assigned to one of the four conditions. The final two schools (both Catholic boys' schools) provided two classes each, and were combined to provide the final block. Within each block of four classes, teachers focused on the same activity content for each of the three lessons in this study (i.e., 
familiarization, baseline, and follow-up). These activities included dance ( $\mathrm{n}=1$ block, 4 classes), netball ( $\mathrm{n}=1$ block, 4 classes), and touch rugby ( $\mathrm{n}=2$ blocks, 8 classes).

\section{Blinding}

Research assistants, blinded to the study hypotheses and condition allocation, collected all data. Students were blinded to allocation; however, teachers were aware of their allocation to an intervention or the control condition.

\section{Statistical methods}

Alpha was set at $p<0.05$ for all tests. We first checked all variables for normality. We then employed MANOVA to examine baseline differences on the primary and secondary outcomes between study completers and those who were lost to follow-up.

To test the fidelity of the interventions, we then conducted two ANCOVAs, one for each item's scores. In these analyses, the post-intervention score was the dependent variable, with the baseline score as the covariate and the condition as the independent variable.

Students also completed the 'choice provided' and 'relevance explained' items. To determine if students also perceived the effects of the intervention on teachers' behavior we used linear mixed models with unstructured covariance matrix to compare intervention and control arms on the two items.

Finally, to examine the effects of the interventions on the primary and secondary outcomes, we also employed mixed model analyses. Specifically, we assessed the fixed effects of experimental condition (control vs. three intervention conditions), time (baseline vs. post-intervention) and a condition-by-time interaction on each variable. All models were analyzed using an intention-to-treat principle, meaning that data from all participants, regardless of whether or not they completed post-intervention assessments, were included in the analyses. In order to account for the clustered nature of the data, school and class were included as random effects in each mixed model. For all analyses, we conducted post-hoc ttest comparisons $(p<.05)$. Standardized mean changes (i.e., Cohen’s $d$; 1988) were calculated, with values of 0.2 (small), 0.5 (moderate), 0.8 (large) used as guidelines for interpreting effect sizes.

\section{Results}

Preliminary Analysis 
An overview of the participant flow through the study can be viewed in Figure 1. Twenty school principals were invited to join the study. Six schools responded favorably, but one eventually declined because of an injury to a PE teacher. Nine schools declined to participate due to time constraints, and five schools did not respond to the study invitation. From the five participating schools, there were 357 students enrolled in Year 8 classes; 288 provided written informed consent from their parents and completed baseline assessment during a PE lesson (80.67\% response rate).

Of the 288 participants who provided data at baseline, 43 did not complete postintervention assessment (15\% loss to follow-up). MANOVA indicated no significant differences on baseline outcome assessments between those who completed post-intervention assessments and those who dropped out of the study (Wilk's $\lambda=.96, F[10,277]=1.08, p=$ .38). None of the variables' distribution significantly departed from normality (skewness and kurtosis, $p<.05)$. All questionnaire subscales produced internally consistent scores at baseline $(\alpha>$.77) and post-intervention $(\alpha>.84)$.

\section{Intervention Fidelity}

\section{Blinded raters}

ANCOVA indicated that for choice provision there was a significant difference between conditions post-intervention, $F(3,11)=11.26, p<.01$. Post-hoc tests revealed that teachers in the providing choice and free choice conditions were rated higher than teachers in the relevance and the control condition $(p<.05)$. These results indicated that both the providing choice and free choice interventions had successfully manipulated expert raters' perceptions of choice provided during the lesson. For the 'relevance explained' item scores, ANCOVA indicated a significant difference between conditions post-intervention, $F(3,11)=$ 5.89, $p=.01$. Teachers in the relevance condition were rated higher than teachers in the other three conditions $(p<.05)$. These results indicated that the relevance intervention successfully manipulated the expert raters’ perceptions of relevance provision during lessons.

\section{Student Ratings}

As shown in Table 1, there was a significant condition-by-time interaction on the choice provided item $(F[6,566]=3.11, p<.01)$. Post-hoc analyses indicated that the change in choice provided score from baseline to post-intervention was greater in the free choice and providing choice conditions than the control and relevance conditions $(p<.01)$. The 
condition-by-time interaction for the relevance explained item was not significant ( $p=.27)$. However, scores in the relevance condition did show significant increase from baseline to post-intervention $(+.73, p<.01, d=.35)$, while scores in all other conditions did not change significantly over time. Overall, these findings supported the fidelity of the intervention as students perceived the expected changes in teacher behavior following the interventions; the choice manipulation may have been more clearly perceived than the relevance manipulation.

\section{Outcomes}

\section{Behavioral Outcomes}

As shown in Table 2, there was a significant condition-by-time interaction on the proportion of time spent in MVPA $(F[3,236.21]=3.14, p=.03)$. Post hoc analyses indicated that the change in MVPA from baseline to post-intervention was greater $(p<.05)$ in the free choice condition ( $+5.79 \%$ of lesson time, $d=.45$ ) compared to the control and the other intervention conditions. Neither of the other intervention conditions showed a significant difference in MVPA change compared with the control condition.

There was a significant condition-by-time interaction on the proportion of lesson time spent sedentary $(F[3,240.79]=9.02, p<.01)$. Post hoc analyses indicated that sedentary time did not change from baseline to post-intervention in the control or relevance conditions. There was a significant reduction in sedentary time from baseline to post-intervention in the providing choice condition (-3.40\% of lesson time, $p=.03, d=-.25$ ); however, this change was not significantly different $(p=.39)$ from the control condition. The free choice condition showed a significant reduction in sedentary time $(-9.90 \%$ of lesson time, $p<.01, d=.72)$ and this change was significantly larger than observed in any of the other conditions $(p<.01)$.

\section{Motivational Outcomes}

There was no significant condition-by-time interaction on the motivation (SDI) scores (see Table 3). The fixed effect of condition was also not significant, but there was a significant time effect $(p<.01)$. Overall, the mean SDI score decreased by a small to moderate amount $(M=-2.07, S E=.31, d=-.39)$ from baseline to post-intervention (across conditions).

There was a significant condition-by-time interaction on autonomy scores $(p=.04)$. Post-hoc testing indicated that autonomy did not change from baseline to post-intervention in 
the control or relevance conditions, but significant increases were observed in the providing choice (+.48, $p<.01, d=.39)$ and free choice (+.37, $p=.02, d=.29)$ conditions. The increase in autonomy found in the providing choice condition was significantly greater than the change in the control condition $(p=.02)$. The difference between the change in autonomy observed in the free choice group was marginally different $(p=.07)$ from the change observed in the control condition.

The condition-by-time interaction for competence scores was marginal ( $p=.08)$. Post-hoc testing indicated that students in the control condition reported increased competence from baseline to post-intervention ( $+.33, p=.03, d=.25)$, while competence did not change in the students in the other conditions $(p>.24)$. The increase in competence in control condition students was significantly greater than the change in the relevance condition $(p=.04)$ and free choice condition $(p=.02)$, but did not differ from the change in the providing choice condition $(p=.29)$.

The interaction of condition-by-time on relatedness scores was not significant $(p=$ .66). Also, there were no significant main effects of time or condition $(p>.45)$ on relatedness scores.

\section{Discussion}

The purpose of this study was to examine the effect of three motivational teaching strategies (i.e., explaining relevance, providing choice, and free choice) on Year 8 students' MVPA levels and motivation during PE lessons. Overall, findings provided support for the effectiveness of the two choice-based strategies.

The providing choice intervention condition successfully increased students' perceived autonomy and decreased students' sedentary behaviors during PE lessons. This finding is in line with previous evidence that providing structured choice from a limited number of options will enhance autonomy (Katz and Assor, 2007; Taylor et al., 2009). The decreases in sedentary time could be surprising to some as the logistical and time demands associated with supporting autonomy, such as giving more instructions and distributing more equipment (Taylor et al., 2009), could have been expected to create more opportunities for students to be sedentary. However, this concern was not supported by our results and, therefore, choice provision appears to be viable strategy to increase student autonomy and decrease sedentary time. 
The free-choice teaching strategy led to greater MVPA and less sedentary behavior, when compared with usual practice, providing choice, and relevance-focused teaching strategies. This finding is supported by Lonsdale and colleagues' (2009) study in which students' pedometer-measured PA levels were greater in the free choice condition than in structured lessons. Taken together, these findings indicate that it is important to integrate free choice activities into PE lessons (Halas, 2004). However, it is important to highlight that PE classes have multiple outcomes that extend beyond PA, including motor skill development, psychosocial regulation, and general health education (Monti, 2004). These learning outcomes may not be fostered effectively in free choice environments. Also, for choice to be beneficial as a long-term motivational strategy, it may need to be accompanied by structure (i.e., the provision of clear expectations and feedback; Jang et al., 2010).

The explaining relevance intervention strategy was not successful in changing any of the behavioral or motivational outcomes. This finding is inconsistent with SDT tenets and experimental evidence, which suggests that adaptive behavior change is likely to occur when the meaning of an activity is highlighted and internalized by the individual (Deci and Ryan, 2000). It also contradicts research indicating that relevance may be more important than choice when promoting student motivation towards academic subjects (Assor et al., 2004). We provided teachers with a number of examples 'relevance' statements, but did not place restrictions on the way they implemented these principles. As such, it is possible that teachers chose relevance statements that may not have been personally meaningful enough for students to promote internalization (i.e., enhanced self-determination). For example, discussing the importance of physical activity for preventing lifestyle diseases may not be personally relevant for adolescents. Moreover, the benefits of emphasizing the relevance and meaning of an activity are strongest when combined with a number of autonomy supportive strategies (Chatzisarantis et al., 2012; Su and Reeve, 2011).

In contrast to our hypotheses, students' autonomous motivation did not increase in any of the teaching strategy conditions. It seems likely that the time span was too small to see any meaningful changes in motivation as a result of the interventions. However, there is substantial evidence that autonomy (which was positively influenced by both choice strategies) is associated with increases in autonomous motivation over time (Edmunds, 2007). Thus, it is plausible that the choice strategies employed in this study could have positive long-term effects on students' motivation. It was also noteworthy that autonomous motivation actually decreased from baseline to post-intervention in all four conditions, including usual practice. This result suggests that students' interest in a particular activity may decrease 
across the course of a unit of study, which often, but not necessarily, focuses on a single activity or sport. Thus, including variety within a unit may be an important strategy to enhance motivation (Dimmock et al., 2013). For example, a unit on invasion games could include a variety of sports (e.g., soccer, lacrosse, and field hockey), rather than spending a number of classes on a single activity.

\section{Limitations}

Several limitations are also noteworthy. The short-term nature of the intervention and post-intervention assessment highlight that although students’ perceived autonomy can change quickly as a result of motivational teaching strategies, but interventions designed to enhance autonomy support may take longer to influence students' motivation (Cheon et al., 2012). Hence, further studies are needed to examine longer-term strategy effects on behavior in, and motivation towards, PE lessons. Additionally, further research is needed to examine intervention effects on objectively measured MVPA outside of PE classes (Chatzisarantis and Hagger, 2008; Cheon et al., 2012). It is also important to acknowledge that generalizability of our findings to other schools could be limited by the modest sample size and by potential selection bias stemming from the use of only schools whose principals were among the first to volunteer to participate. Also, we did not collect data concerning the proportion of time in each lesson spent on specific activities (e.g., teacher speaking to students vs. students completing skill building drills vs. students playing modified games). These different activities may provide varying opportunities for students to engage in MVPA. In the future, researchers may wish to collect these data and investigate if any systematic differences in specific activities occur as a result of the implementation of different motivational strategies. Finally, we anticipated ICCs of 0.1 for our primary outcomes (Rosenkranz et al., 2012), yet the observed clustering for MVPA was much larger (ICC = 0.62). This unexpected result is similar to a recent study in school PE (Aelterman et al., 2012) and likely reduced our power to detect significant effects on this variable. However, sensitivity analysis conducted without 
adjusting for clustering (i.e., no random effects included) produced highly similar findings to the results presented in this report (contact the first author for details).

\section{Conclusion}

Physical education programs have the potential to positively impact children and adolescents' health by providing opportunities to accumulate PA. However, current PA levels in PE lessons are often insufficient to provide these benefits. Promoting choice can produce short-term increases in PA and decreases in sedentary behavior. Greater emphasis on choicebased motivational teaching strategies in PE teacher preparation programs and school curricula could help to increase the amount of PA that students accumulate. 


\section{References}

Aelterman, N., Vansteenkiste, M., Van Keer, H., Van den Berghe, L., De Meyer, J., Haerens, L., 2012. Students' objectively measured physical activity levels and engagement as a function of between-class and between-student differences in motivation toward physical education. J. Sport Exerc. Psychol. 34:457.

Assor, A., Kaplan, H., Roth, G., 2002. Choice is good, but relevance is excellent: Autonomyenhancing and suppressing teacher behaviours predicting students engagement in schoolwork. Br. J. Educ. Psychol. 72:261-78.

Assor, A., Roth, G., Deci, E.L., 2004. The emotional costs of parents' conditional regard: A self-determination theory analysis. J. Pers. 72:47-88.

Campbell, M.K., Elbourne, D.R., Altman, D.G., 2004. CONSORT statement: extension to cluster randomised trials. BMJ 328:702-08.

Centers for Disease Control and Prevention, 2010. Strategies to improve the quality of physical education., Washington, DC.

Chatzisarantis, N.L., Hagger, M.S., Kamarova, S., Kawabata, M., 2012. When effects of the universal psychological need for autonomy on health behaviour extend to a large proportion of individuals: a field experiment. British journal of health psychology 17:785-97. Chatzisarantis, N.L.D., Hagger, M.S., 2008. Effects of an intervention based on selfdetermination theory on self-reported leisure-time physical activity participation. Psychology \& Health 24:29-48.

Cheon, S.H., Reeve, J., Moon, I.S., 2012. Experimentally based, longitudinally designed, teacher-focused intervention to help physical education teachers be more autonomy supportive toward their students. J. Sport Exerc. Psychol. 34:365-96.

Cohen, J., 1988. Statistical power analysis for the behavioral sciences, 2nd ed. Lawrence Erlbaum, Hillsdale, N.J.

Crawford, D., 2009. The future of sport in Australia. Commonwealth of Australia, Canberra. Deci, E.L., Ryan, R.M., 2000. The "what" and "why" of goal pursuits: Human needs and the self-determination of behavior. Psychological Inquiry 11:227-68.

Dimmock, J., Jackson, B., Podlog, L., Magaraggia, C., 2013. The effect of variety expectations on interest, enjoyment, and locus of causality in exercise. Motivation and Emotion:1-8. 
Edmunds, J., 2007. Adherence and well-being in overweight and obese patients referred to an exercise on prescription scheme: A self-determination theory perspective. Psychology of Sport and Exercise 8:722-40.

Fairclough, S., Stratton, G., 2005. Physical activity levels in middle and high school physical education: A review. Pediatr. Exerc. Sci. 17:217.

Fairclough, S.J., Stratton, G., 2006. A review of physical activity levels during elementary school physical education. Journal of Teaching in Physical Education 25:239-57.

Freedson, P.S., Pober, D., Janz, K.F., 2005. Calibration of accelerometer output for children. Med. Sci. Sports Exerc. 37:523-30.

Gillison, F.B., Standage, M., Skevington, S.M., 2013. The effects of manipulating goal content and autonomy support climate on outcomes of a PE fitness class. Psychol. Sport Exerc. 14:342-52.

Guay, F., Vallerand, R.J., Blanchard, C., 2000. On the assessment of situational intrinsic and extrinsic motivation: The Situational Motivation Scale (SIMS). Motiv. Emotion 24:175-213. Hagger, M.S., Chatzisarantis, N., Barkoukis, V., Wang, C.K.J., Baranowski, J., 2005. Perceived autonomy support in physical education and leisure-time physical activity: A cross-cultural evaluation of the trans-contextual model. J. Educ. Psychol. 97:376-90. Halas, J., 2004. Questioning our assumptions: Unconventional lessons from the "Swamp of Practice". Journal of Physical Education, Recreation \& Dance 75:14-21.

Hardy, L.L., King, L., Espinel, P., Cosgrove, C., Bauman, A., 2010. NSW Schools Physical Activity and Nutrition Survey (SPANS): Full report, Sydney: NSW Ministry of Health. Jaakkola, T., Liukkonen, J., Laakso, T., Ommundsen, Y., 2008. The relationship between situational and contextual self-determined motivation and physical activity intensity as measured by heart rates during ninth grade students' physical education classes. European Physical Education Review 14:13.

Jang, H., Reeve, J., Deci, E.L., 2010. Engaging students in learning activities: It is not autonomy support or structure but autonomy support and structure. J. Educ. Psychol. 102:588-600.

Katz, I., Assor, A., 2007. When choice motivates and when it does not. Educational Psychology Review 19:429-42.

Lonsdale, C., Rosenkranz, R.R., Peralta, L.R., Bennie, A., Fahey, P., Lubans, D.R., 2013. A systematic review and meta-analysis of interventions designed to increase moderate-tovigorous physical activity in school physical education lessons. Prev. Med.:152-61. 
Lonsdale, C., Sabiston, C.M., Raedeke, T.D., Ha, A.S., Sum, R.K., 2009. Self-determined motivation and students' physical activity during structured physical education lessons and free choice periods. Prev. Med. 48:69-73.

Lonsdale, C., Sabiston, C.M., Taylor, I.M., Ntoumanis, N., 2011. Measuring student motivation for physical education: Examining the psychometric properties of the Perceived Locus of Causality Questionnaire and the Situational Motivation Scale. Psychology of Sport and Exercise 12:284-92.

McAuley, E., Duncan, T., Tammen, V.V., 1989. Psychometric properties of the Intrinsic Motivation Inventory in a competitive sport setting: a confirmatory factor analysis. Res. Q. Exerc. Sport 60:48.

Monti, B., 2004. Appropriate practices for high school physical education. National Association for Sport and Physical Education, an association of the American Alliance for Health, Physical Education, Recreation and Dance.

Ng, J.Y.Y., Lonsdale, C., Hodge, K., 2011. The Basic Needs Satisfaction in Sport Scale (BNSSS): Instrument development and initial validity evidence. Psychology of Sport and Exercise 12:257-64.

Office for Standards in Education Children’s Services and Skills, 2013. Beyond 2012 outstanding physical education for all: Physical education in schools. , Manchester, UK. Patall, E.A., Cooper, H., Robinson, J.C., 2008. The effects of choice on intrinsic motivation and related outcomes: A meta-analysis of research findings. Psychol. Bull. 134:270-300. Perlman, D.J., 2013. The influence of the social context on students' in-class physical activity Journal of Teaching in Physical Education 32:46-60.

Richer, S.F., Vallerand, R.J., 1998. Construction et validation de l'Echelle du Sentiment d’Appartenance Sociale (ESAS). Revue Européenne de Psychologie Appliquée/European Review of Applied Psychology 48:129-37.

Rosenkranz, R.R., Lubans, D.R., Peralta, L.R., Bennie, A., Sanders, T., Lonsdale, C., 2012. A cluster-randomized controlled trial of strategies to increase adolescents' physical activity and motivation during physical education lessons: the Motivating Active Learning in Physical Education (MALP) trial. BMC Public Health 12:834.

Ryan, R.M., Deci, E.L., 2002. Overview of self-determination theory: An organismic dialectical perspective, in: Deci, E.L., Ryan, R.M. (Eds.), Handbook of self-determination research. The University of Rochester Press, Rochester, NY, pp. 3-33. 
Standage, M., Duda, J.L., Ntoumanis, N., 2003. A model of contextual motivation in physical education: Using constructs from self-determination and achievement goal theories to predict physical activity intentions. J. Educ. Psychol. 95:97.

Standage, M., Gillison, F., 2007. Students’ motivational responses toward school physical education and their relationship to general self-esteem and health-related quality of life. Psychology of Sport and Exercise 8:704-21.

Su, Y.L., Reeve, J., 2011. A meta-analysis of the effectiveness of intervention programs designed to support autonomy. Educational Psychology Review 23:159-88.

Taylor, I.M., Lonsdale, C., 2010. Cultural differences in the relationships among autonomy support, psychological need satisfaction, subjective vitality, and effort in British and Chinese physical education. J. Sport Exerc. Psychol. 32:655-73.

Taylor, I.M., Ntoumanis, N., Smith, B., 2009. The social context as a determinant of teacher motivational strategies in physical education. Psychology of sport and exercise 10:235-43. Trost, S.G., Loprinzi, P.D., Moore, R., Pfeiffer, K.A., 2011. Comparison of accelerometer cut points for predicting activity intensity in youth. Med. Sci. Sports Exerc. 43:1360-68.

US Department of Health and Human Services, 2008. Physical Activity Guidelines Advisory Committee Report, Washington, DC.

Vansteenkiste, M., Simons, J., Soenens, B., Lens, W., 2004. How to become a perservering exerciser? Providing a clear, future intrinsic goal in an autonomy-supportive way. Journal of Sport and Exercise Psychology 26:232-49.

Ward, J., Wilkinson, C., Graser, S.V., Prusak, K.A., 2008. Effects of Choice on Student Motivation and Physical Activity Behavior in Physical Education. Journal of Teaching in Physical Education 27:385-98. 